\title{
Designing of spherical chitosan nano-shells with micellar cores for solvation and safeguarded delivery of strongly lipophilic drugs
}

\author{
Esra Cihan ${ }^{\mathrm{a}}$, Mehmet Polat ${ }^{\mathrm{b}}$, Hurriyet Polat ${ }^{\mathrm{a}, *}$ \\ a Izmir Institute of Technology, Department of Chemistry, Urla, Izmir, Turkey \\ b Izmir Institute of Technology, Department of Chemical Engineering, Urla, Izmir, Turkey
}

\section{H I G H L I G H T S}

- Dissolving and transporting strongly lipophilic drugs in body fluids is a challenge.

- Highly hydrophobic drug Probucol was dissolved in the micellar cores of a copolymer.

- Chitosan was polymerized as a shell around the micelles containing the dissolved drug.

- The chitosan nano-shells were wellformed, spherical and $30-300 \mathrm{~nm}$ in diameter.

- They preserve the micelle integrity and provide a $\mathrm{pH}$-sensitive release environment.

\section{A R T I C L E I N F O}

\section{Keywords:}

Micelle(s)

Chitosan

Nanoparticle(s)

Nano-shell(s)

Lipophilic drug(s)

\section{G R A P H I C A L A B S T R A C T}
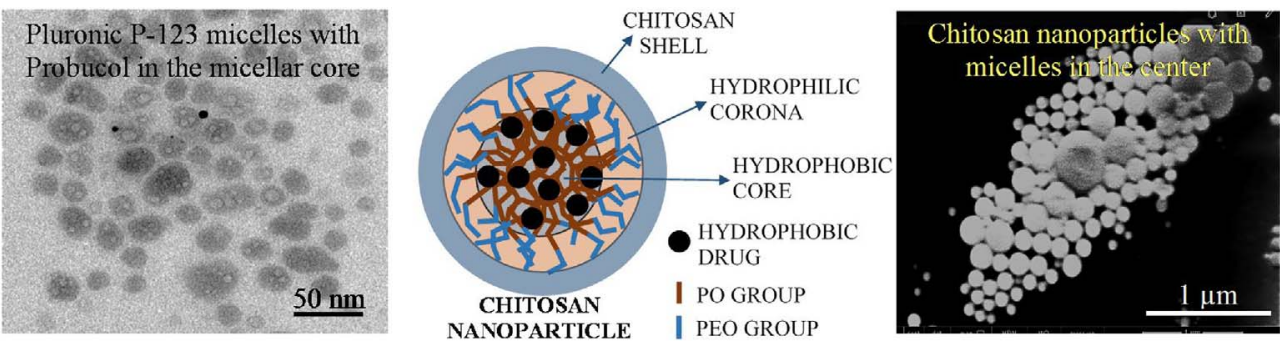

\footnotetext{
* Corresponding author.

E-mail address: hurriyetpolat@iyte.edu.tr (H. Polat).
} 


\section{Introduction}

Natural polymers such as cellulose, starch, chitosan, carrageenan, alginates etc. are among the preferred materials for drug delivery applications due to their chemically inert, nontoxic, biocompatible, biodegradable structures and availability. Among these materials, chitosan-based nanoparticles have been receiving special attention due to their superior characteristics as i) high drug-carrying capacities, ii) multi-functionality, ii) prolonged circulation and iv) favorable targeting and penetrability of the cell membranes $[1-8,10]$. Grenha (2012) presents an excellent review of the methods for manufacturing chitosan nanoparticles which can be grouped into the following categories: emulsification and cross-linking [8,9], emulsion droplet coalescence [11,12], emulsion solvent diffusion [13], reverse micellization [14-17], ionic gelation [3,18-21], polyelectrolyte complexation [22-34], modified ionic gelation with radical polymerization $[23,35,36]$ and desolvation [37-40]. Despite this wide research base, complications still exist in the synthesis of the chitosan nanoparticles due to their aversion for uptaking and encapsulating lipophilic drug active materials and the difficulties in controlling their size and morphology.

This study describes a reproducible and effective method to manufacture spherical, hollow, well-defined chitosan nanoparticles with a micellar core which functions as a solvent for a completely water-insoluble drug. Due to this unique shape, the nanoparticles will also be termed shelled nanoparticles or nano-shells interchangibly throughout the text. The method comprises of two distinct stages which provide superior control in both the drug loading and morphological properties of the manufactured particles. Loading of the drug and creation of the soft templates for subsequent particle formation are achieved in the first stage. It is based on dissolution and encapsulation of the lipophilic drug molecules within the hydrophobic cores of the micelles of a PEO-PPOPEO type three-block copolymer. The choice of the copolymer for this paper was Pluronic P-123, but these polymeric surfactants, in general, provide excellent opportunities for adjusting the solvation characteristics due to their wide HLB range which can be exploited to vary the concentration and density of the drug molecules loaded. Polymerization of chitosan by cross-linking over the drug-loaded micelles to form perfectly spherical nano-sized particles constitutes the second stage. This stage provides outstanding flexibility for controlling the size and morphology of the final particles and modifying their surface functionality.

\section{Materials and methods}

\subsection{Materials}

A Pluronic type triblock copolymer (P-123) with relatively longer hydrophobic blocks $\left(\mathrm{PEO}_{20}-\mathrm{PPO}_{65}-\mathrm{PEO}_{20}\right)$ was employed to create the template micelles. This copolymer was preferred due to its commercial availability, biocompatibility, and safety [41,42].

The drug Probucol (PB) was employed for loading of the micelles. Probucol is an anti-hyperlipidemic drug which functions by lowering the cholesterol level in the blood by inhibiting cholesterol synthesis and delaying cholesterol absorption. It is a white, odorless crystalline powder with a melting point of $125^{\circ} \mathrm{C}$. It is freely soluble in chloroform, benzene, ether, acetone, ethanol, methanol and acetonitrile; but completely insoluble in water. It must be stressed that selection of probucol was solely due to its strong lipophilic characteristic in order to demonstrate that a completely water-insoluble drug active material could be embedded within chitosan.

The other chemicals employed in the study were acetonitrile (AN) for co-solving the drug with the block copolymer, sodium dodecyl sulfate (SDS) for modification of the micelles, acetic acid (AA) for chitosan dissolution and sodium tripolyphosphate (TPP) for chitosan polymerization. All the chemicals were supplied by SIGMA-ALDRICH chemical company and their most basic properties are presented in
Table 1. Ultra-pure water (18.2 M $\Omega$ ) was used throughout the study.

The carbon grids and the mica surfaces for the transmission and atomic force microscopy characterization work were provided by "Micro to Nano" Innovative Microscopy Supplies Netherlands/(ValueTec-TEM-support-grids).

\subsection{Method}

The chitosan was precipitated in the form of a shell around the surfactant micelles which were previously loaded with the lipophilic drug. Hence, the procure consisted of two stages: the thin-film hydration step where the micelles were loaded with the drug and the ionic gelation step where the chitosan was polymerized around the micelles. A generalized flowsheet of the study is presented in Fig. 1.

In the initial phase, the P-123 micelles were dissolved together with the water-insoluble drug in acetonitrile. Surface tension (ST) measurements were employed to ensure that the P-123 molecules were in the form of nearly spherical micelles. Subsequent evaporation of the solvent generated an organic composite film of the polymer and the drug. Hydration of this film in an aqueous phase forces the lipophilic drug molecules energetically into the hydrophobic core of the micelles. In this study, the aqueous phase was a solution of chitosan $(0.5 \%$ by weight) in an acetic acid solution ( $0.5 \%$ by volume). In some cases, the micelles of P-123 were modified by SDS in order to control the size and charge of the micellar structures. The aggregation number of the P-123 micelles is around 50 [43]; substitution of the relatively neutral P-123 structures with the smaller SDS molecules should lead to a compaction of the micelle while introducing a negative charge [44]. A carbon grid was dipped into an aliquot of the micellar solution and was dried and kept under vacuum to prepare a representative sample for the were transmission electron microscopy (TEM) characterization of the micelles. The same procedure was repeated with a mica surface into the micellar solution to prepare samples for the atomic force microscopy (AFM) characterization of the micelles.

In the final phase, the micellar chitosan solution was reacted with a cross-linking agent (TPP; $0.5 \%$ by weight) at a chitosan-TPP ratio of $1: 1$ to polymerize chitosan around the micelles as a shell. The presence of acetic acid in the system ensures that the solution $\mathrm{pH}$ is maintained around 4. Cross-linking of the chitosan molecules with TPP takes place through the negatively charged oxygens in the TPP with the positively charged amine groups on chitosan (Fig. 2) [45]. The precipitated particles are centrifuged and dried directly without any washing in vacuum under ambient conditions. A schematic view of the chitosan nano-shells manufactured is illustrated in Fig. 3. The figure shows only one micelle in the structure; however, more than one micelle can also be enveloped by the chitosan cell.

Prior to the characterization studies, the amount of the probucol which was enveloped within the chitosan structure was estimated by measuring the amount of probucol in the centrifuge supernatant with UV-vis spectroscopy [46]. The dried chitosan nanoparticles were characterized using scanning transmission electron microscopy (STEM), dynamic light scattering (DLS), dynamic light scattering with laser velocimetry (DLS-LV), Fourier transform infrared spectroscopy (FTIR). A list of all the characterization methods employed in this study is summarized in Table 2.

For the preliminary drug release tests, the dried particles were dispersed $(0.4 \%$ by weight) in simulated body fluids [47] of different $\mathrm{pH}$ values of 6.8 and 7.4 and the amount of drug released into the solution was checked using UV-vis spectroscopy $[46,48]$.

\section{Results and discussion}

\subsection{Characterization of the P-123 micelles}

Fig. 4 gives the results of surface tension measurements of P-123 solutions. The surface tension measurements were performed to 
Table 1

Basic properties of the materials and chemicals employed in the study.

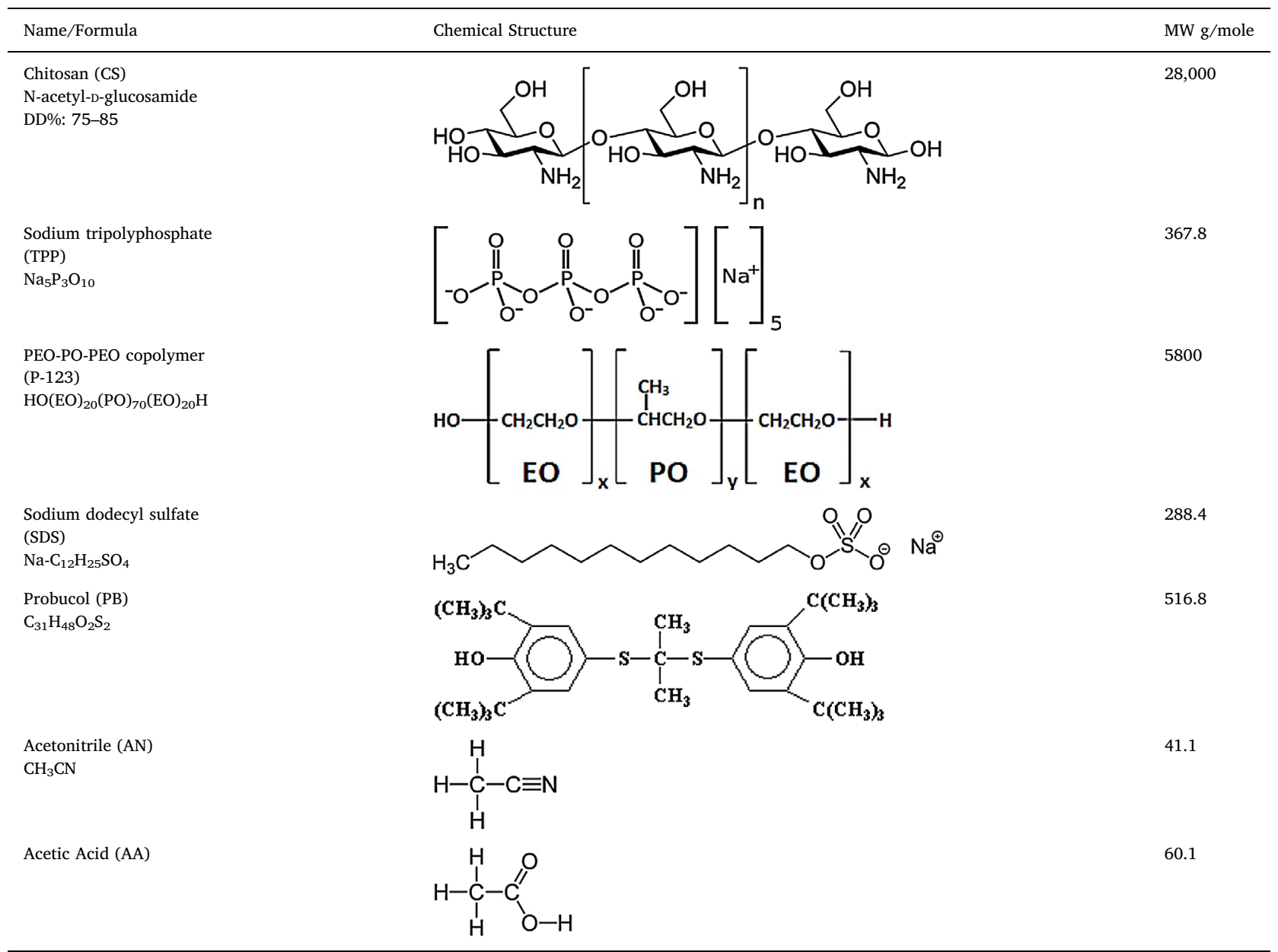

determine the association behavior of the P-123 molecules in aqueous solution. The graph demonstrates that the surface tension-concentration profiles of the P-123 solutions could roughly be divided into 3 distinct regions: In Region I, the surface tension decreases steadily with increasing concentration. In this region, the molecules of the copolymer are in monomer form. Region II is a transition region marked by a shallower slope indicating the initiation of aggregation where the copolymer molecules start to form dimers, trimers, etc. progressively. The molecules aggregate in the form of full grown micelles in Region III and the surface tension remains constant with changes in concentration. The CMC value of P-123 could be determined from the interception between Regions II and III and it was found to be around $5 \times 10^{-5} \mathrm{M}$.

Fig. 5 presents the three repeat size distributions of the P-123 micelles by dynamic light scattering at a concentration of $10^{-3} \mathrm{M}$. The reason for selecting this concentration is the fact that it is well-past the CMC but low enough to generate tight, spherical since a transition from spherical to cylindrical form begins to take place at around $10^{-2} \mathrm{M}$ [49]. It can be seen from the graph that the micelle size varies between 8 and $40 \mathrm{~nm}$. The $d_{50}$ median size for the distribution is around $20 \mathrm{~nm}$.

The size and shape of the micelles at this concentration were also investigated using atomic force microscopy and scanning transmission electron microscopy and the images obtained are presented in Fig. 6. The results of the AFM and STEM imaging confirm the average micelle size indicated by DLS. The images also demonstrate that the micelles formed were closer to a spherical shape as expected.

\subsection{Modification of the P-123 micelles by SDS}

It has been shown previously that addition of smaller, anionic surfactant molecules into a solution of P-123 resulted in mixed micelles via hydrophobic association of the surfactant with the block copolymer micelles resulting in smaller and negatively charged copolymer micelles (Bhattacharjee, 2013). In this study, the surfactant employed for the modification of the micelle size of the copolymer P-123 was the simple anionic surfactant sodium dodecyl sulfate (SDS). It was confirmed that the solubilization of the hydrocarbon chains of the smaller SDS into the polypropylene oxide core of the P-123 resulted in a steady decrease in the size of the micelles with an increase in the concentration of the anionic surfactant. The method provides the means of controlling the size of the chitosan nano-shells which are formed around the copolymeric surfactant micelles. Some representative size measurement results obtained with DLS are presented in Fig. 7 showing the size distribution of the micelles of pure SDS, pure P-123, and SDS + P-123 mixture. It can be seen that the median size of the micelles of the anionic SDS was much smaller, around $1.5 \mathrm{~nm}$ compared to the median size of $20 \mathrm{~nm}$ for the P-123 micelles. However, when the anionic surfactant was mixed to the copolymer, the micelles of the mixed system were significantly smaller with a median size of around $5 \mathrm{~nm}$. This suggests that the SDS molecules were incorporated into the structure of the P-123 micelles and resulted in a more compact structure. The lack of a bimodal distribution for the SDS + P-123 mixture is indicative of the fact that the SDS molecules were not present as separate micelles but were incorporated into the micellar structure of the P-123 copolymer. 


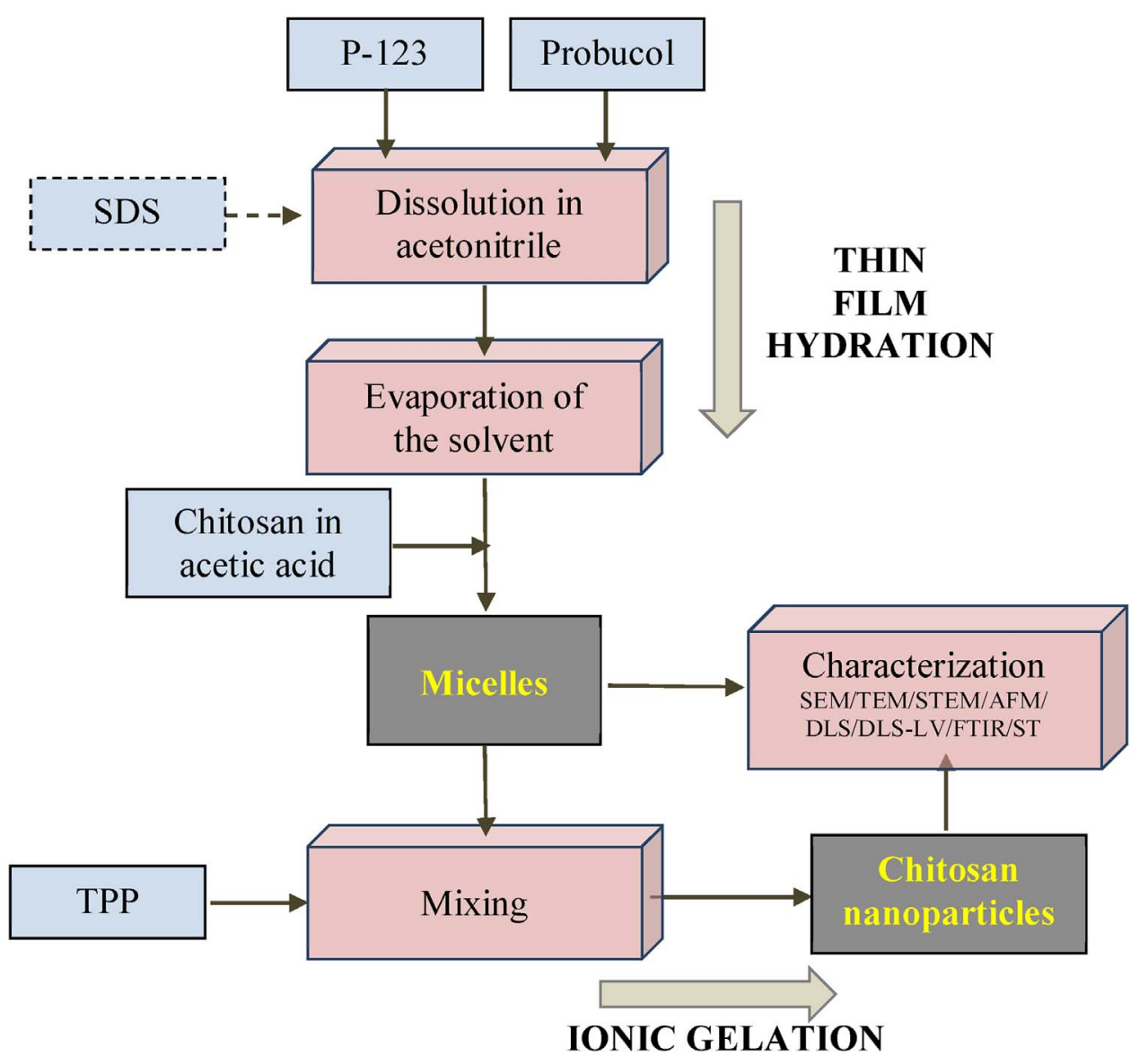

Fig. 1. Flowsheet of the method used to create chitosan nanoparticles embedded with P-123 micelles encapsulating the lipophilic drug in the micellar core.
The zeta potentials of the micelles under the same conditions were determined by DLS-LV and are presented in Fig. 8 for the same solutions presented in Fig. 7. The figure shows that the micelles of the anionic SDS were charged negatively with a median zeta potential of around $-32 \mathrm{mV}$. The micelles of the P-123 were charged to a much less degree with a median zeta potential of around $-12 \mathrm{mV}$. The surface charge of the mixed micelles of the binary SDS + P-123 mixture was negatively charged with a median zeta potential of around $-22 \mathrm{mV}$, confirming that the SDS molecules were incorporated into the structures of the P123 micelles.

\subsection{Characterization of the drug-loaded P-123 micelles}

The P-123 micelles were loaded with a lyophilic drug probucol by thin-film hydration method described above. Characterization of the drug-loaded micelles was done by STEM and TEM. The STEM images of

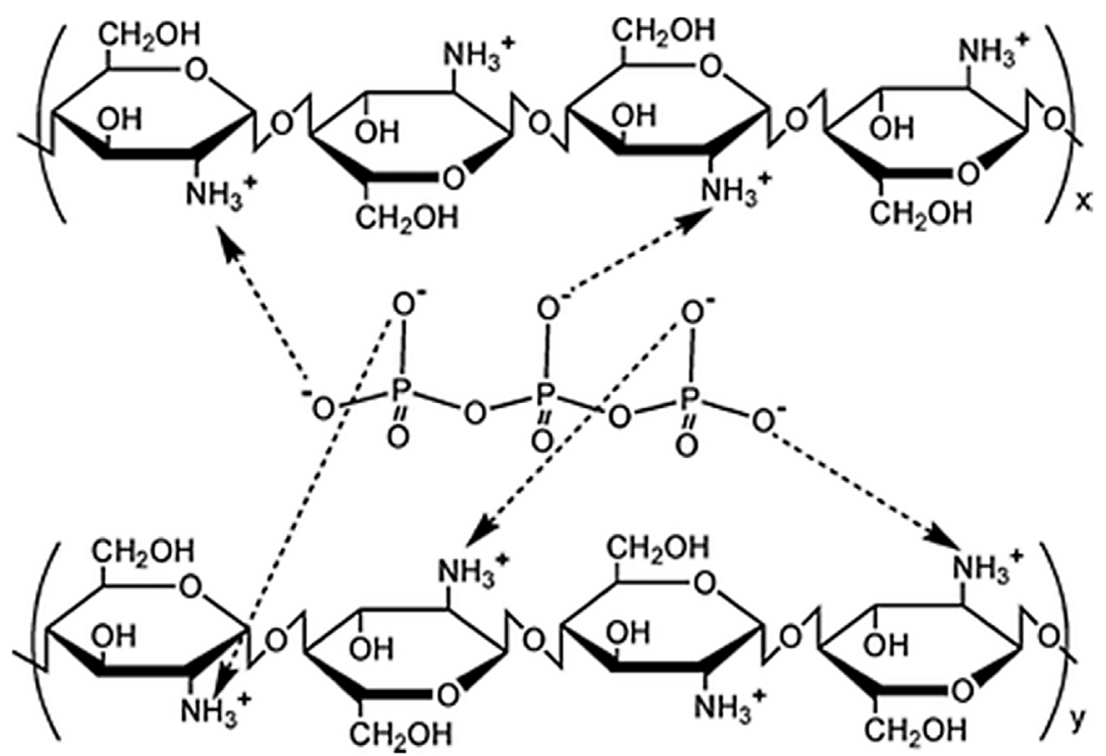

Fig. 2. The mechanism of cross-linking of chitosan by TPP. 


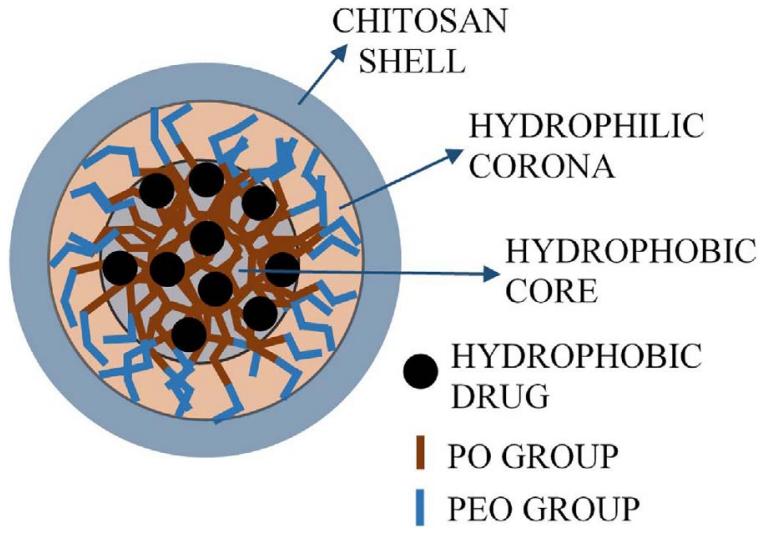

Fig. 3. The schematic structure of a micelle-embedded chitosan nanoparticle.

Table 2

Characterization methods used in this study.

\begin{tabular}{lll}
\hline Analysis & Method & Device \\
\hline Micellar Size & DLS & Malvern Zetasizer Nano ZS \\
Micellar Charge & DLS-LDV & Malvern Zetasizer Nano ZS \\
Micellar morphology & AFM-Tapping mode & Digital Inst. Nanoscope IV \\
Particle morphology & STEM & Quanta 250 SEM \\
Inner morphology & TEM & FEI Tecnai G2 Spirit Bio Twin \\
Particle composition & FTIR & Perkin Elmer \\
Surface tension & Dü Noüy Ring & Krüss Tensiometer K10ST \\
Drug Release & UV-vis & Varian Cary 50 UV \\
\hline
\end{tabular}

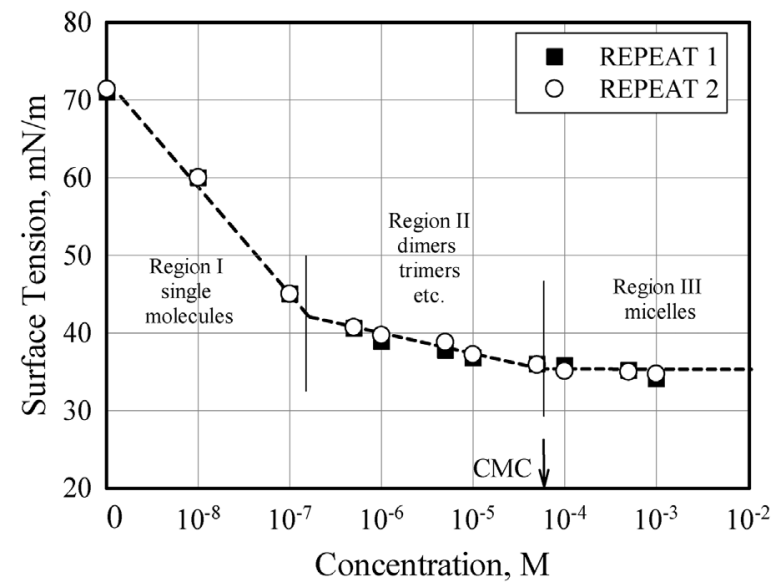

Fig. 4. Surface tension data for the aqueous solutions of P-123.

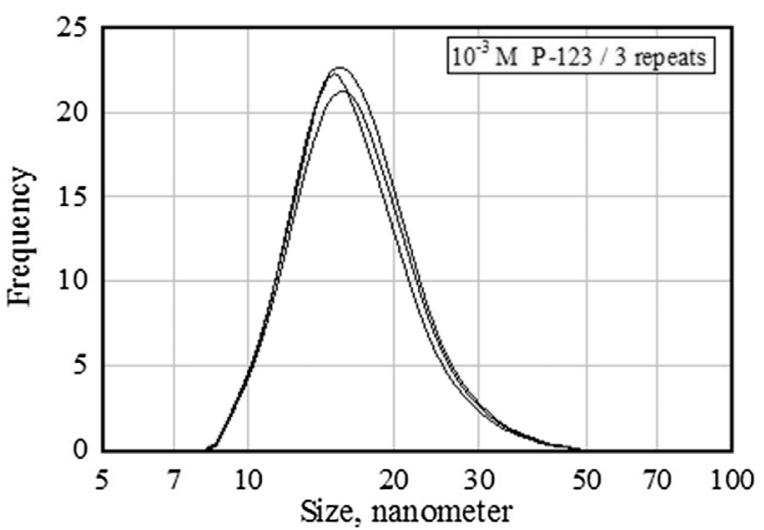

Fig. 5. Size distribution of the P-123 micelles in 10-3 M aqueous P-123 solutions.
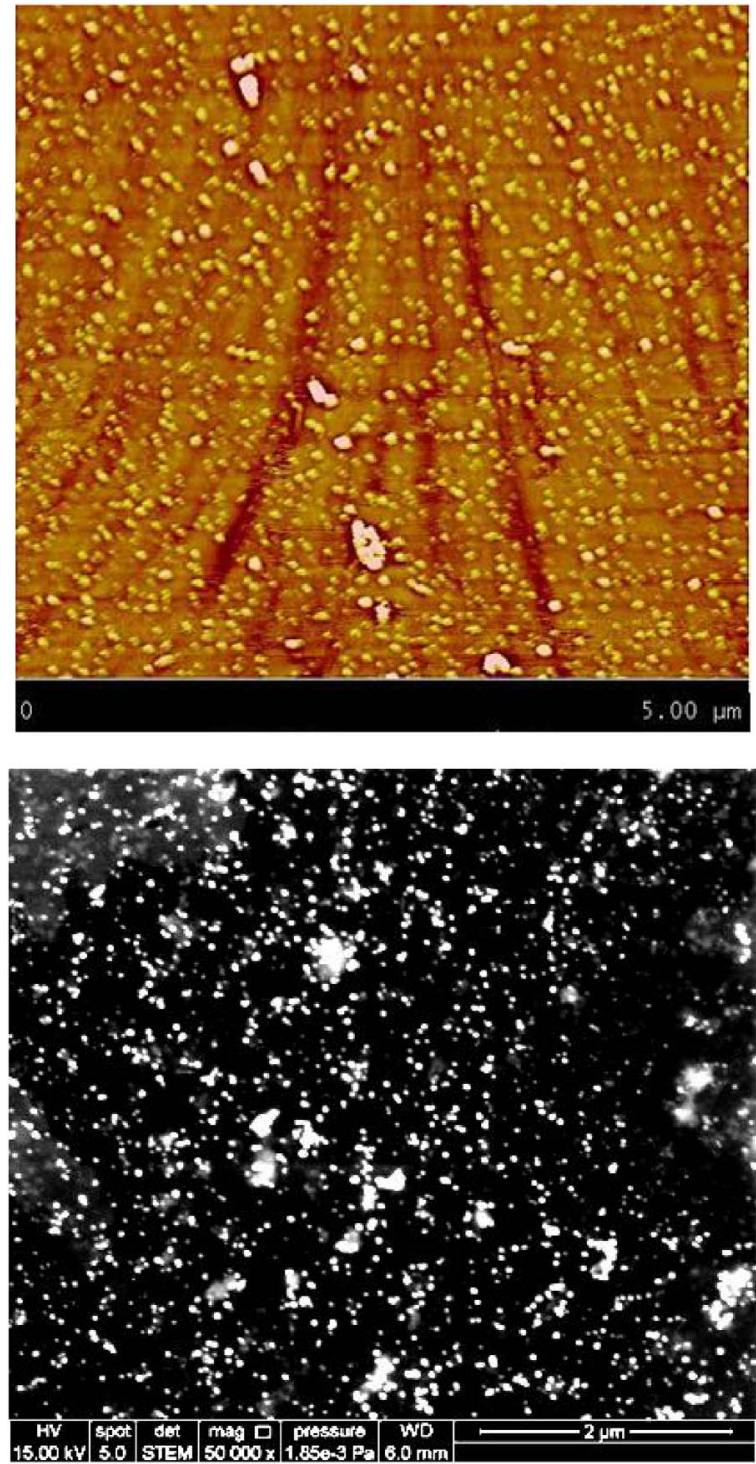

Fig. 6. The AFM (top) and STEM (bottom) images showing the size and morphology of the P-123 micelles at 10-3 M aqueous P-123 solution.

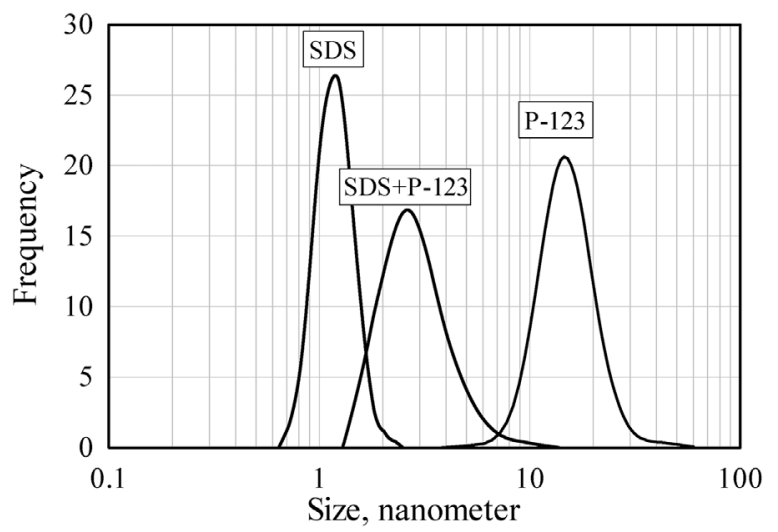

Fig. 7. Size distribution of the micelles of aqeous SDS, P-123 and SDS + P-123 solutions.

the drug-loaded P-123 micelles are presented in Fig. 9 (right) along with the images of the blank P-123 micelles in the absence of probucol (left) for comparison. A more detailed TEM image of the drug-loaded P123 micelles are presented in Fig. 10.

The STEM images clearly show that the size of the P-123 micelles 


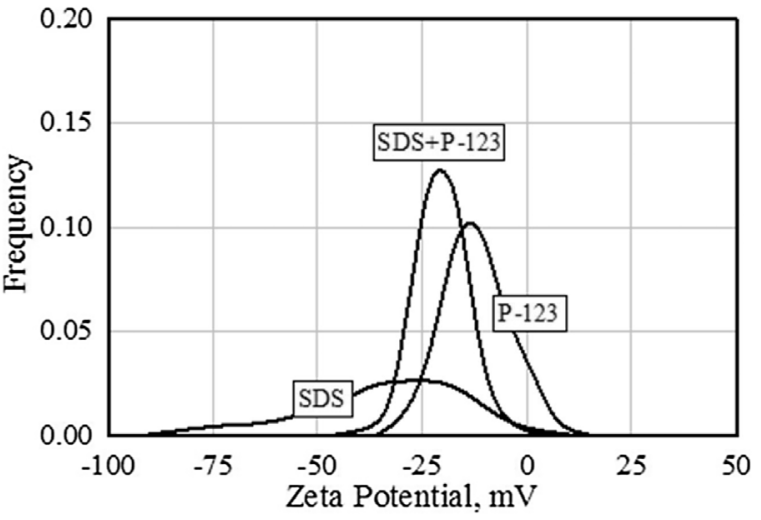

Fig. 8. Zeta Potential distribution of the micelles of aqeous SDS, P-123 and SDS + P-123 solutions.

increases significantly when the drug probucol is loaded into the micelle structure. The STEM images also demonstrate that the spherical shape of the micelles is preserved after the loading of the drug. Also, the relatively narrow size distribution of the micelles becomes wider when the drug is incorporated into the micellar structure. The TEM images of the loaded micelles display the drug in the micellar structure very clearly and confirm both the shape and the range of sizes for the micelles generated in the AFM, STEM and DLS studies.

\subsection{Characterization of the hollow chitosan nanoparticles (nano-shells) encapslating the probucol-loaded P-123 micelles}

In this part of the study, the lipophilic drug probucol was incorporated into the chitosan structure by cross-linking the chitosan around the drug-loaded formed micelles using the ionic gelation procedure. The final product was in the form of nanoparticles which comprised of a chitosan shell around the P-123 micelles which contain probucol dissolved in the hydrophobic core (see Fig. 3). The particles synthesized were characterized by STEM, FTIR, and DLS-LDV analyses. The CS/TPP ratio, CS\%, and TPP solution percent were kept constant at unity in the manufacturing of the particles subjected to these analyses though they could be varied to manipulate the size of the nanoparticles produced. The STEM images of the shelled chitosan nanoparticles are presented in Fig. 11. The figure shows that chitosan particles of spherical shape and excellent consistency could be produced successfully. It can be observed from the figure that the size of the chitosan particles ranged from 30 to $300 \mathrm{~nm}$. The presence of 30 nanometer particles indicate that some chitosan shells envelope only a single micelle while the relatively wide size distribution suggests that in some cases chitosan may have precipitated around more than one loaded P-123 micelle.

The zeta potentials of the chitosan nano-shells precipitated around the loaded P-123 micelles were measured and presented in Fig. 12 together with the zeta potential of the chitosan particles cross-linked

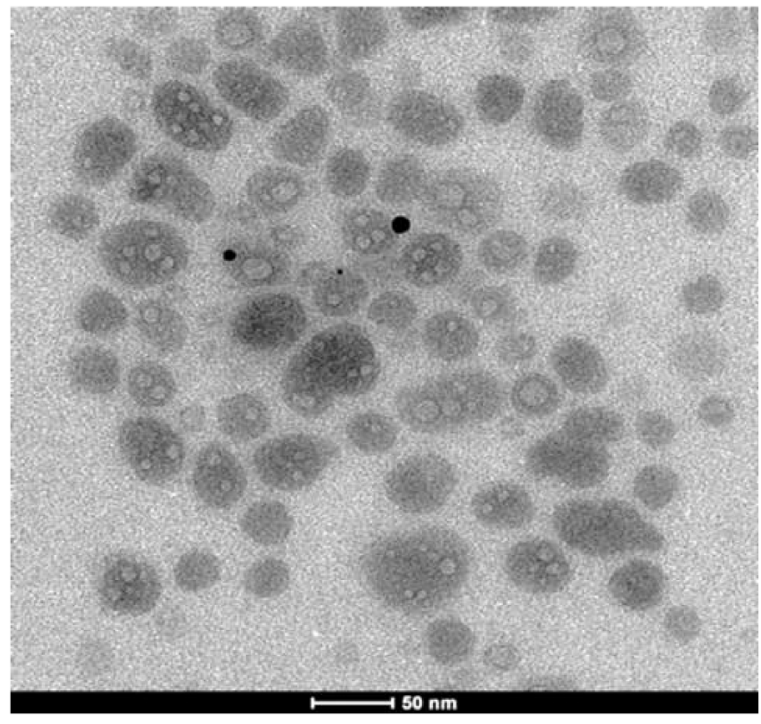

Fig. 10. TEM images of the P-123 micelles loaded with the drug probucol.

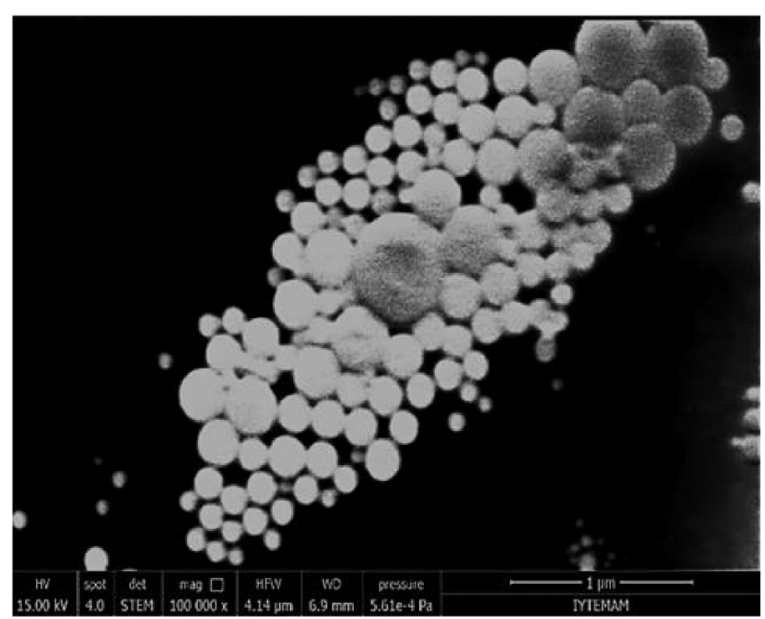

Fig. 11. The STEM images of the shelled chitosan nanoparticles (nano-shells) loaded with the P-123 micelles containing the dissolved Probucol.

without any copolymer or drug in the system. The precipitated particles were washed and dispersed in distilled water prior to the zeta potential measurements. The two charge distributions are almost very similar, indicating that the micelles of the copolymer P-123 were enveloped within the chitosan shells to a large degree.

The structure of the chitosan nanoparticles with enveloped micelles which contained probucol was further investigated by FTIR analysis. The FTIR spectrum of the chitosan nanoparticles is presented together

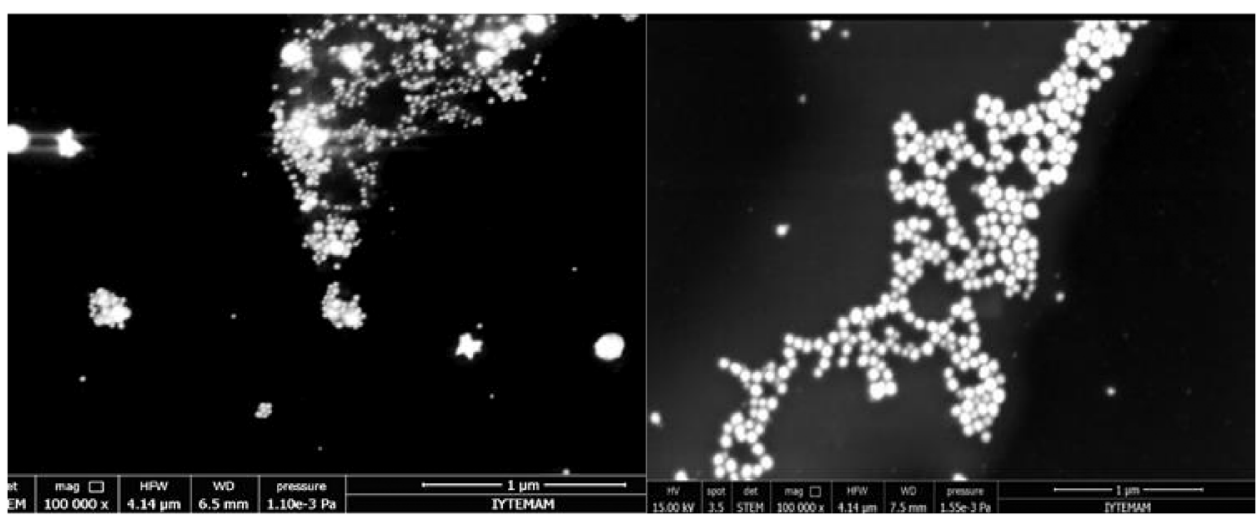

Fig. 9. The STEM images of the P-123 micelles without (left) and loaded with the drug probucol (right) in the structure at the same magnification. 


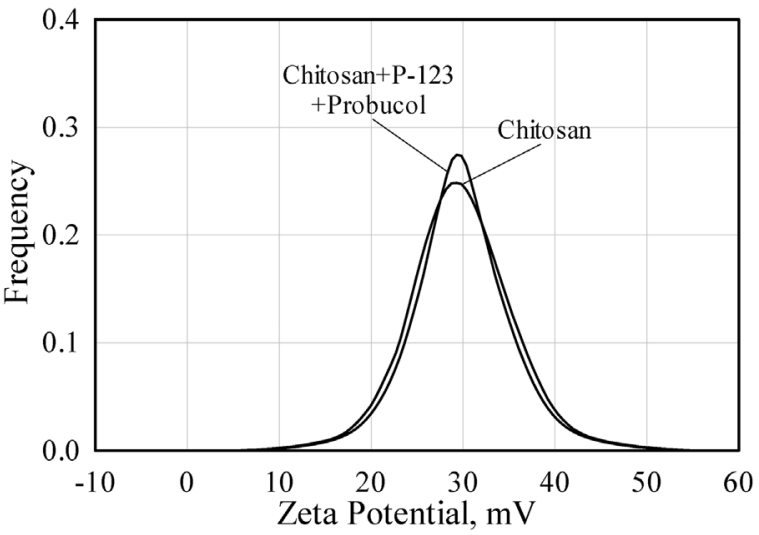

Fig. 12. Zeta potential measurements of the chitosan nano-shells with and without the P123 micelles.

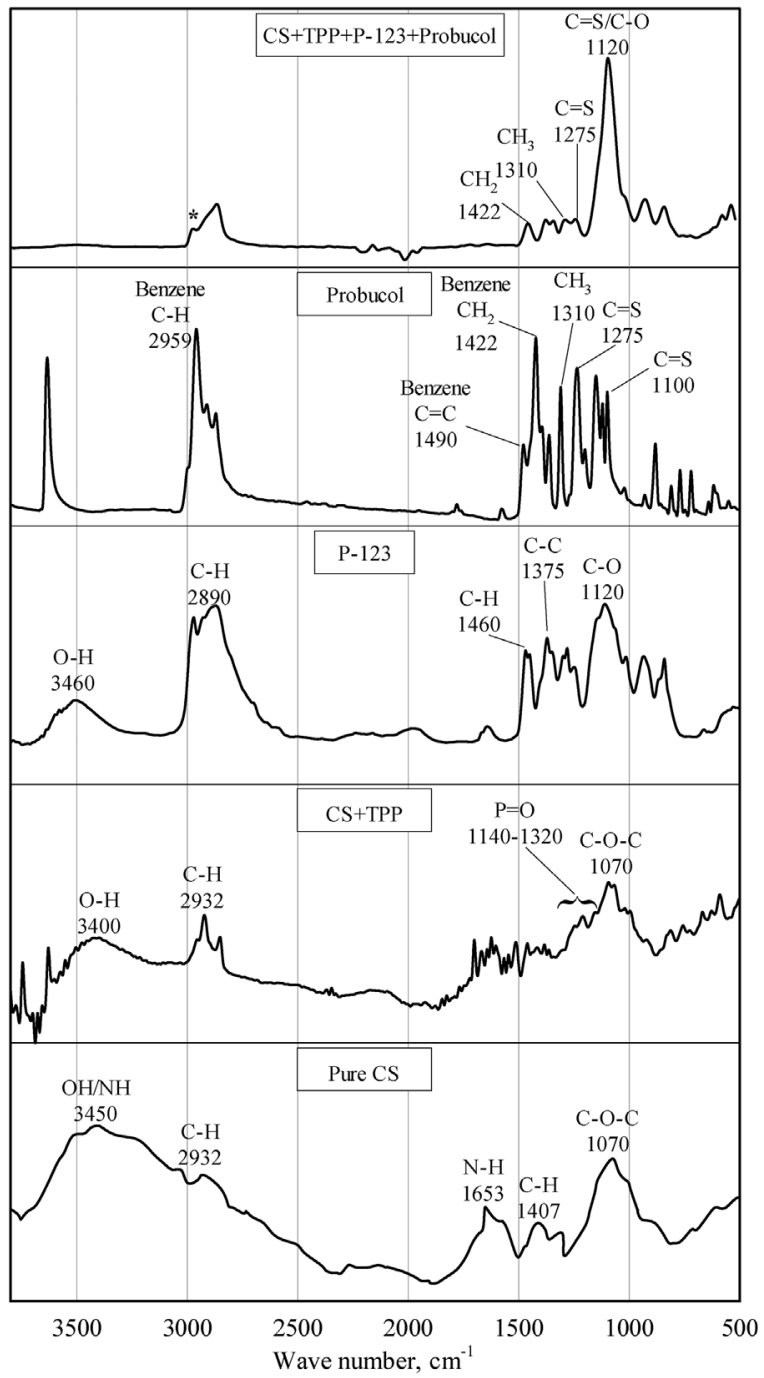

Fig. 13. FTIR results of the chitosan nanoparticles embedded with the drug loaded P- 123 micelles.

with the spectrums of the pure chitosan (Pure CS), cross-linked chitosan (CS + TPP), pure P-123 and pure probucol in Fig. 13 for comparison. The characteristic peaks obtained for pure forms of chitosan, P-123 and probucol were in very good agreement with the spectra in the literature [50-54].

The absorption bands at 1653 and $3450 \mathrm{~cm}^{-1}$ are ascribed to $\mathrm{N}-\mathrm{H}$ bending in the primary amine of pure chitosan. The intensity of these peaks decreases significantly after polymerization with TPP most probably due to the linkage between tri polyphosphoric groups of TPP and ammonium groups of chitosan. Though it is not presented in Fig. 13 for clarity, TPP also has a very sharp characteristic peak for the P-O-P band at $892 \mathrm{~cm}^{-1}$. The disappearance of this band in the CS + TPP system is another indication of successful cross-linking between the negatively charged oxygen and the positively charged ammonium groups (see Fig. 2). Furthermore, the appearance of the $\mathrm{P}=\mathrm{O}$ band at $1140-1250 \mathrm{~cm}^{-1}$ in the chitosan + TPP system which is non-existent in the pure chitosan spectra is yet another indication since doublebonded oxygen does not make any bonds with the chitosan.

Probucol and P-123 spectra show several sharp, overlapping characteristic peaks in the $500-1500$ and $2800-3000 \mathrm{~cm}^{-1}$ regions which complicate the analysis since the appearance of these peaks in the spectra of the micelle-loaded chitosan nano-shells can mean either P123 or probucol is in the structure. However, some peaks which appear in the spectra of the nanoparticles are specific to either P-123 or probucol. For example, the peaks at 2890, 1375, 1120 and $910 \mathrm{~cm}^{-1}$ are specific only to P-123. Similarly, the strong peaks at 1422, 1310 and $1275 \mathrm{~cm}^{-1}$ are specific to probucol. The fact that all these peaks are present in the spectra of the loaded chitosan nano-shells clearly indicate that not only P-123, but also probucol is present in the structure.

\subsection{The uptake of probucol and preliminary release tests}

Prior to the release studies, the amount of the probucol which was enveloped within the chitosan structure was estimated by deducting the amount of probucol in the centrifuge supernatant from the original probucol amount in the system [46]. The uptake in two repeat tests was found to be very high; $86.7 \%$ and $93.4 \%$ of the initial probucol amount. The release tests were carried out with a known amount of dried chitosan particles dispersed ( $0.4 \%$ by weight) in simulated body fluid [47] of different $\mathrm{pH}$ values $(\mathrm{pH} 6.8$ and 7.4). These $\mathrm{pH}$ values were representative of the acidic environment of cancerous and healthy cells, respectively [55]. The amount of drug released into the solution was checked using UV-vis spectroscopy.

Two repeat tests were carried out at the specified $\mathrm{pH}$ values and the results are presented in Fig. 14. It can be seen that the release is very $\mathrm{pH}$-specific. Under the $\mathrm{pH}$ conditions predominant with the healthy cells, a release of about $10 \%$ takes place within the first hour and no more drug release is observed. The reason for this initial release is most probably due to the release of the residual micelle-probucol structures which have not been enveloped within the chitosan shells but remained in the solution and clung to the surfaces of the chitosan particles during centrifuging and drying step since no washing was applied to the centrifuged particles. The same initial release was also observed at the acidic $\mathrm{pH}$ value of 6.8 , but it was slightly higher at around $12 \%$. The reason for the slightly higher release was most probably due to the

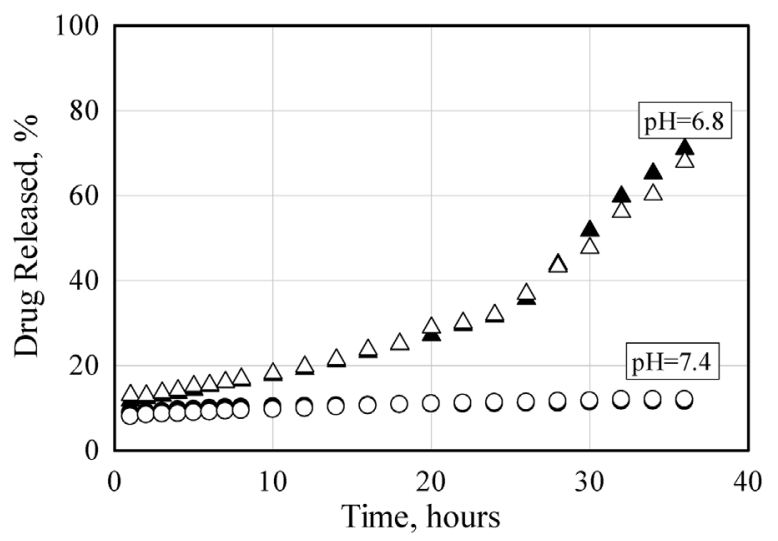

Fig. 14. The preliminary drug release data showing the release of the drug probucol from the chitosan nanoparticles dispersed in the simulated body fluid at two different $\mathrm{pH}$. 
additional release of the drug from inside the chitosan particles due to the initiation of chitosan degradation at this acidic $\mathrm{pH}$. The release continued in an accelerated fashion and reached $75 \%$ after $36 \mathrm{~h}$. The difference in the release profiles between $\mathrm{pH} 6.8$ and $\mathrm{pH} 7.4$ is very drastic and demonstrates simultaneously the presence of the drug within the chitosan nano-shells as well as the potential of the shelled chitosan nanoparticles as $\mathrm{pH}$ sensitive, target specific delivery vehicles for drug release.

\section{Conclusions}

In this study, a strongly lipophilic drug (Probucol) was embedded inside hollow, spherical, nano-sized chitosan particles (nano-shells) by combining the thin-film hydration and ionic gelation techniques. The procedure was based on first dissolving the drug in the hydrophobic core of the micelles of a biocompatible copolymer (P-123) by ionic gelation and subsequent cross-linking of the chitosan molecules around the micellar soft-templates by ionic gelation. The product was wellformed, hollow and perfectly spherical chitosan nanoparticles (nanoshells) in the size range of $30-300 \mathrm{~nm}$.

The manufactured nanoparticles were characterized using scanning transmission and transmission electron microscopy, atomic force microscopy, dynamic light scattering, dynamic light scattering with laser velocimetry, Fourier transform infrared spectroscopy and surface tension analyses. The study has shown that:

- the strongly lipophilic drug probucol was successfully dissolved in the cores of the micelles of a PEO-PPO-PEO triblock copolymer (Pluronic P-123) using thin-film hydration method.

- Polymerization of the chitosan around the micelles by ionic gelation allowed incorporating this lipophilic drug into the structure of chitosan successfully. The final product was in the form of wellformed, nano-sized, spherical and hollow chitosan particles

- the procedure provides a very good control on the size and morphology of the particles manufactured as well as controlling the amount of the drug loading.

- The drug uptake by the chitosan nanoparticles was very high. Two repeat tests demonstrated that $86.7 \%$ and $93.4 \%$ of the initial probucol amount was incorporated into the chitosan structure.

- The preliminary release tests demonstrated that there was no appreciable release at $\mathrm{pH} 7.4$ where the integrity of the chitosan shells should be preserved. The drastic increase in the release amounts at the acidic $\mathrm{pH}$ of 6.8 proved simultaneously the presence of the drug within the particles as well as their potential as $\mathrm{pH}$ sensitive, target specific delivery vehicles for drug release.

\section{References}

[1] T.M. Fahmy, P.M. Fong, A. Goyal, W.M. Saltzman, Targeted for drug delivery, Mater. Today 8 (2005) 18-26.

[2] L.E. Gerweck, Tumor pH: implications for treatment and novel drug design, Semin. Radiat. Oncol. 8 (1998) 176-182.

[3] P. Calvo, C. Remunan-Lopez, J.L. Vila-Jato, J. Alonso, The potential of chitosan for pulmonary drug delivery, J. Appl. Polym. Sci. 63 (1997) 125-132.

[4] X. Zou, X. Zhao, L. Ye, Q. Wang, H. Li, Preparation and drug release behavior of pHresponsive bovine serum albumin-loaded chitosan microspheres, J. Ind. Eng. Chem. 21 (2015) 1389-1397.

[5] F. Chen, Y. Zhu, Chitosan enclosed mesoporous silica nanoparticles as drug nanocarriers: sensitive response to the narrow $\mathrm{pH}$ range, Microporous Mesoporous Mater. 150 (2012) 83-89.

[6] J.P. Jee, J.H. Na, S. Lee, S.H. Kim, K. Choi, Y. Yeo, I.C. Kwon, Cancer targeting strategies in nanomedicine: design and application of chitosan nanoparticles, Curr. Opin. Solid State Mater. Sci. 16 (2012) 333-342.

[7] Q. Yuan, R. Subramanian, S. Hein, R.D.K. Misra, Stimuli-responsive magnetic nanoparticle drug carrier: magnetite encapsulated with chitosan grafted-copolymer, Acta Biomater. 4 (2008) 1024-1037.

[8] Q. Yuan, J. Shah, S. Hein, R.D.K. Misra, Controlled and extended drug release behavior of chitosan-based nanoparticle carrier, Acta Biomater. 6 (2010) 1140-1148.

[9] Y. Ohya, M. Shiratani, H. Kobayashi, T. Ouchi, Release behaviour of 5-fluorouracil from chitosan-gel nanospheres immobilizing 5-fluorouracil coated with polysaccharides and their cell specific cytotoxicity, Pure Appl. Chem. A31 (1994)
629642.

[10] Z. Songjiang, W. Lixiang, Amyloid-Beta associated with chitosan nano-carrier has favorable immunogenicity and permeates the BBB, AAPS PharmSciTech 10 (2009) 900-905.

[11] H. Tokumitsu, H. Ichikawa, Y. Fukumori, L.H. Block, Chem Preparation of gadopentetic acidloaded chitosan microparticles for gadolinium neutron-capture therapy of cancer by a novel emulsion-droplet coalescence technique, Pharm. Bull. 47 (1999) 838-842.

[12] S.M. Anto, C. Kannan, K.S. Kumar, S.V. Kumar, M. Suganeshwari, Formulation of 5fluorouracil loaded chitosan nanoparticles by emulsion droplet coalescence method for cancer therapy, Int. J. Pharm. Biol. Arch. 2 (2011) 926-931.

[13] M.H. El-Shabouri, Positively charged nanoparticles for improving the oral bioavailability of cyclosporin-A, Int. J Pharm. 249 (2002) 101-108.

[14] S. Mitra, U. Gaur, P.C. Ghosh, A.N. Maitra, Tumor targeted delivery of encapsulated dextrandoxorubicin conjugate using chitosan nanoparticles as carriers, J. Controlled Release 74 (2001) 317-323.

[15] T. Banerjee, S. Mitra, A.K. Singh, R.K. Sharma, A. Maitra, Preparation, characterization and biodistribution of ultrafine chitosan nanoparticles, Int. J. Pharm. 243 (2002) 93-105.

[16] Z.-X. Tang, J.-Q. Qian, L.-E. Xi, Appl. Biochem, Preparation of chitosan nanoparticles as carrier for immobilized enzyme, Biotechnol 136 (2007) 77-96.

[17] R. Manchanda, R. Nimesh, Controlled size chitosan nanoparticles as an efficient, biocompatible oligonucleotides delivery system, J. Appl. Polym. Sci. 118 (2010) 2071-2077.

[18] A. De Campos, A. Sanchez, M.J. Alonso, Chitosan nanoparticles: a new vehicle for the improvement of the delivery of drugs to the ocular surface. Application to cyclosporin A, Int. J. Pharm. 224 (2001) 159-168.

[19] Z. Ma, H.H. Yeoh, L.-Y. Lim, Formulation pH modulates the interaction of insulin with chitosan nanoparticles, J. Pharm. Sci. 91 (2002) 1396-1404.

[20] J. Wang, J.D. Byrne, M.E. Napier, J.M. DeSimone, More effective nanomedicines through particle design, Small 7 (2011) 1919-1931.

[21] W. Fan, W. Yan, Z. Xu, H. Ni, Formation mechanism of monodisperse, low molecular weight chitosan nanoparticles by ionic gelation technique, Colloid Surf. B: Biointerfaces 90 (2012) 21-27.

[22] P. Erbacher, S. Zou, T. Bettinger, A.-M. Steffan, J.-S. Remy, Chitosan-based vector/ DNA complexes for gene delivery: biophysical characteristics and transfection ability, Pharm. Res. 15 (1998) 1332-1333.

[23] Y. Hu, X. Jiang, Y. Ding, H. Ge, Y. Yuan, C. Yang, Synthesis and characterization of chitosanpoly(acrylic acid) nanoparticles, Biomaterials 23 (2002) 31933201.

[24] J. Du, R. Sun, S. Zhang, T. Govender, L.F. Zhang, C.D. Xiong, Y.X. Peng, Novel polyelectrolyte carboxymethyl konjac glucomannan-chitosan nanoparticles for drug delivery, Macromol. Rapid Commun. 25 (2004) 954-958.

[25] B. Sarmento, A. Ribeiro, F. Veiga, D. Ferreira, Development and characterization of new insulin containing polysaccharide nanoparticles, Colloid Surf. B: Biointerfaces 53 (2006) 193-202.

[26] B. Sarmento, A. Ribeiro, F. Veiga, D. Ferreira, R. Neufeld, Oral bioavailability of insulin contained in polysaccharide nanoparticles, Biomacromolecules 8 (2007) 3054-3060.

[27] Y.-H. Lin, F-L. Mi, C.-T. Chen, W-C. Chang, S.-F, Peng, H.-F, Liang, H.-W. Sung, Preparation and characterisation of nanoparticles shelled with chitosan for oral insulin delivery, Biomacromolecules 8 (2007) 146-152.

[28] A. Bayat, B. Larijani, S. Ahmadian, H.E. Junginger, M. Rafiee-Tehrani, Preparation and characterization of insulin nanoparticles using chitosan and its quaternized derivatives, Nanomed. Nanotechnol. Biol. Med. 4 (2008) 115-120.

[29] Y.-H. Lin, K. Sonaje, K.M. Lin, J.-H. Juang, F.-L. Mi, H.-W. Yang, H.-W. Sung, Multiioncrosslinked nanoparticles with $\mathrm{pH}$-responsive characteristics for oral delivery of protein drugs, J. Controlled Release 132 (2008) 141-149.

[30] D. Teijeiro-Osorio, C. Remuñan-López, M.J. Alonso, Biomacromolecules 10 (2009) 243-249.

[31] M.R. Avadi, A.M.M. Sadeghi, N. Mohammadpour, S. Abedin, F. Atyabi, R.M. Dinarvand, Rafiee-Tehrani, Preparation and characterization of insulin nanoparticles using chitosan and arabic gum with ionic gelation method, Nanomed. Nanotechnol. Biol. Med. 6 (2010) 58-63.

[32] A. Grenha, M.E. Gomes, M. Rodrigues, V.E. Santo, J.F. Mano, N.M. Neves, R.L. Reis, Development of new chitosan/carrageenan nanoparticles for drug delivery applications, J. Biomed Mater. Res. 92A (2010) 1265-1272.

[33] S. Kaihara, Y. Suzuki, K. Fujimoto, In situ synthesis of polysaccharide nanoparticles via polyion complex of carboxymethyl cellulose and chitosan, Colloid Surf. B: Biointerfaces 85 (2011) 343-348.

[34] M.K. Yeh, K.M. Cheng, C.S. Hu, Y.C. Huang, J.J. Young, Novel protein-loaded chondroitin sulfate-chitosan nanoparticles: preparation and characterization, Acta Biomater. 7 (2011) 3012-3804.

[35] S. Sajeesh, C.P. Sharma, Cyclodextrin-insulin complex encapsulated polymethacrylic acid based nanoparticles for oral insulin delivery, Int. J. Pharm. 325 (2006) 147-154.

[36] S. Sajeesh, C.P. Sharma, Novel pH responsive polymethacrylic acid-chitosan-polyethylene glycol nanoparticles for oral peptide delivery, J. Biomed. Mater. Res. 76 B (2006) 298-305.

[37] H.-Q. Mao, K. Roy, V.L. Troung-Le, K.A. Janes, K.Y. Lin, Y. Wang, J.T. August, K.W. Leong, Chitosan-DNA nanoparticles as gene carriers: synthesis, characterization and transfection efficiency, J. Controlled Release 70 (2001) 399-421.

[38] O. Borges, G. Borchard, J.C. Verhoef, A. Sousa, H.E. Junginger, Preparation of coated nanoparticles for a new mucosal vaccine delivery systems, Int. J. Pharm 229 (2005) 155-166.

[39] S.A. Agnihotri, T.M. Aminabhavi, Chitosan nanoparticles for prolonged delivery of timolol maleate, Drug Dev. Ind. Pharm. 33 (2007) 1254-1262. 
[40] F. Atyabi, F. Talaie, R. Dinarvand, Thiolated chitosan nanoparticles as an oral de livery system for amikacin: in vitro and ex vivo evaluations, J. Nanosci. Nanotechnol. 9 (2009) 4593-4603.

[41] A.V. Kabanov, E.V. Batrakova, V.Y. Alakhov, Pluronic block copolymers as novel polymer therapeutics for drug and gene delivery, J. Controlled Release 82 (2002) 189-212.

[42] B. Foster, T. Cosgrove, B. Hammouda, Pluronic triblock copolymer systems and their interactions with ibuprofen, Langmuir 25 (2009) 6760-6766.

[43] P. Alexandridis, A. Hatton, Poly (ethylene oxide)-poly (propylene oxide) block copolymer surfactants in aqueous solutions and at interfaces: thermodynamics, structure, dynamics, and modeling, Colloids Surf. A 96 (1995) 1-46.

[44] J. Bhattacharjee, G. Verma, V.K. Aswal, V. Patravalec, P.A. Hassan, Microstructure drug binding and cytotoxicity of Pluronic P123-aerosol OT mixed micelles, RSC Adv. 3 (2013) 23080-23089.

[45] C.H. Yang, Y.S. Lin, K.S. Huang, Y.C. Huang, E.C. Wang, J.Y. Jhong, C.Y. Kuo, Microfluidic emulsification and sorting assisted preparation of monodisperse chit osan microparticles, Lab Chip 9 (2009) 145-150.

[46] E. Cihan, Design of Micelle Embedded Chitosan Nano Composites for Targeted Delivery of Hydrophobic Drugs MSc Thesis, Izmir Institute of Technology, 2016.

[47] A. Oyane, H.M. Kim, T. Furuya, T. Kokubo, T. Miyazaki, T. Nakamura, Preparation and assessment of revised simulated body fluids, J. Biomed. Mater. Res. A 65 (2003) $188-195$.

[48] L.M.U. Dutra, Rilbeiro M.E.N.P, I.M. Cavalcante, D.H.A. de Brito, L.M. Semiao,
R.F. de Silva, S.G.Yeates P.B.A.Fechine, N.M.P.S. Ricardo, Binary mixtures micellar systems of F127 and P123 for griseofulvin solubilisation, Polimeros 25 (2015) 433-439.

[49] P. Petrov, J. Yuan, K. Yoncheva, A.H.E. Müller, C.B. Tsvetanov, Wormlike morphology formation and stabilization of pluronic P123 micelles by solubilization of pentaerythritol tetraacrylate, J. Phys. Chem. B 112 (2008) 8879-8883.

[50] I. Corazzari, R. Nisticò, F. Turci, M.G. Faga, F. Franzoso, S. Tabasso, G. Magnacca, Advanced physico-chemical characterization of chitosan by means of TGA coupled on-line with FTIR and GCMS: Thermal degradation and water adsorption capacity, Polym. Degrad. Stab. 112 (2015) 1-9.

[51] W. Ajun, S. Yan, G. Li, L. Huili, Preparation of aspirin and probucol in combination loaded chitosan nanoparticles and in vitro release study, Carbohydr. Polym. 75 (2009) 566-574.

[52] R. Sarma, M. Dambarudhar, Luminescence and bio-imaging response of thio-glycolic acid (TGA) and sodium dodecyl sulfate (SDS)-coated fluorescent cadmium selenide quantum dots, J. Lumin. 161 (2015) 395-402.

[53] N. Jindal, S.K. Mehta, Nevirapine loaded Poloxamer 407/Pluronic P123 mixed micelles: optimization of formulation and in vitro evaluation, Colloids Surf. B: Biointerfaces 129 (2015) 100-106.

[54] S. Walke, G. Srivastava, M. Nikalje, J. Doshi, R. Kumar, S. Ravetkar, P. Doshi, Fabrication of chitosan microspheres using vanillin/TPP dual crosslinkers for protein antigens encapsulation, Carbohydr. Polym. 128 (2015) 188-198.

[55] J.R. Griffiths, Are cancer cells acidic? Br. J. Cancer 64 (1991) 425-427. 